Revue d'histoire de l'Amérique française

REVUE D.HISTOIRE DE L'AMÉRIQUE FRANÇAISE

\title{
Régulation sociale et répression de la déviance au Bas-Canada au tournant du $19^{\mathrm{e}}$ siècle (1791-1815)
}

Jean-Marie Fecteau

Volume 38, numéro 4, printemps 1985

URI : https://id.erudit.org/iderudit/304305ar

DOI : https://doi.org/10.7202/304305ar

Aller au sommaire du numéro

Éditeur(s)

Institut d'histoire de l'Amérique française

ISSN

0035-2357 (imprimé)

1492-1383 (numérique)

Découvrir la revue

Citer cet article

Fecteau, J.-M. (1985). Régulation sociale et répression de la déviance au

Bas-Canada au tournant du $19^{\mathrm{e}}$ siècle (1791-1815). Revue d'histoire de

l'Amérique française, 38(4), 499-521. https://doi.org/10.7202/304305ar d'utilisation que vous pouvez consulter en ligne. 


\title{
RÉGULATION SOCIALE ET RÉPRESSION DE LA DÉVIANCE AU BAS-CANADA AU TOURNANT DU 19e SIECLE (1791-1815) ${ }^{1}$
}

\author{
JEAN-MARIE FECTEAU \\ Département d' histoire \\ Université du Québec à Montréal
}

Les carences de la recherche historique sur le système répressif québécois ne sont plus à démontrer. Il n'existe, en fait, aucune étude générale d'envergure en ce domaine ${ }^{2}$. Les quelques études empiriques menées dans ce champ ont été surtout le fait de juristes historiens s'attachant à cerner l'évolution et la perception du droit criminel après la Conquête $^{3}$, si l'on excepte évidemment le Régime français couvert par les recherches d'André Lachance. L'étude du système pénal s'est donc réduite, pour une bonne part, soit à l'analyse du droit en vigueur, soit tout simplement au récit plus ou moins romancé des «affaires criminelles». Pourtant, au-delà du fonctionnement concret des cours et du triste sort de criminels plus ou moins célèbres, c'est le mode de gestion des illégalismes perpétrés par les classes populaires dont il est finalement question.

Une étude scientifique de l'économie répressive assignable à un ensemble social donné - ici, le Québec au tournant du 19e siècle - ne peut faire abstraction du cadre structurel dans lequel s'inscrit cette économie et sa logique sociale d'opération.

\footnotetext{
1 Ce texte s'inspire de la partie de notre thèse de doctorat touchant l'appareil répressif. Voir Jean-Marie Fecteau, La pauvreté, le crime, l'État. Essai sur l'économie politique du contrôle social au Québec, 1791-1840, (Paris, Université de Paris VII, juin 1983), 480 p. Nous tenons à remercier les membres du Groupe québécois de recherche en histoire du contrôle social, et particulièrement André Morel et André Lachance, pour leurs remarques sur une première version de ce texte.

2 Nous faisons abstraction ici des chroniques criminelles publiées par Raymond Boyer, Les crimes et châtiments au Canada français du 17 e au 20 e siècle (Montréal, Cercle du livre de France, 1966), et plus récemment par Hélène-Andrée Bizier, Crimes et châtiments. La petite histoire du crime au Québec (Montréal, Libre Expression, 1982), 2 vols. Pour un survol récent, voir Louis A. Knafla et T. L. Chapman, «Criminal Justice in Canada. A Comparative Study of the Maritimes and Lower Canada, 1760-1812», Osgoode Hall Law Journal, 21 (juin 1983): 245 274.

Sur l'administration de la justice et l'adaptation du droit anglais à la colonie, voir notamment les travaux d'André Morel, «Les crimes et les peines: évolution des mentalités au 19e siècle», Revue de droit, 8 (1977): 384-396; «La réaction des Canadiens devant l'administration de la justice de 1764 à 1774», Revue du barreau de la province de Québec, 20 (1960): 53-63; "La réception du droit criminel anglais au Québec (1760-1892)», Revue juridique Thémis, 13 (1978): 449-541. Voir aussi Jacques L'Heureux, «L'organisation judiciaire au Québec de 1764 à 1774», Revue générale de droit, 1 (1970): 266-331; J.-E. Côté, «The Reception of English Law», Alberta Law Review, 15 (1977): 29-92; Claude Desaulnier, «La peine de mort dans la législation criminelle de 1760 à 1892», Revue générale de droit, 8 (1977): 141-184. Chez les historiens, deux exceptions notables: Hilda Neatby, The Administration of Justice under the Quebec Act (Minnesota, University of Minnesota Press, 1937), et surtout Douglas Hay, «The Meaning of the Criminal Law in Quebec, 1764-1774», dans Louis A. Knafla (ed.), Crime and Criminal Justice in Europe and Canada (Waterloo, Wilfrid Laurier University Press, 1981), 77-111.
} 
En effet, la répression du crime est beaucoup plus qu'une simple opération de police mettant aux prises, de façon plus ou moins atemporelle, la «société» contre le vaste registre des manquements à l'ordre. Sa cible, son organisation et les moyens que cette répression emploie relèvent d'une problématique précise, d'une logique que la science sociale, historique ou autre, doit mettre à jour. Ainsi sont soulevés une série de problèmes dont il est impossible, dans une recherche pertinente du sens des pratiques utilisées, de faire l'économie. Le problème de la «gestion» du crime à une époque donnée débouche donc sur un ensemble d'interrogations fondamentales: quels rapports entretient l'organisation politique - l'État - avec la formation sociale en cause; quelle est la place ou le mode d'articulation du droit dans le système de prise en charge du crime; quel est le rôle assigné à l'appareil institutionnel dans la pratique répressive? C'est à une première tentative de réponse à ces questions fondamentales qu'est consacré ce bref article.

\section{L'État bas-canadien et la répression du crime}

Toute analyse du système répressif à l'oeuvre dans une formation sociale pose immédiatement la question de l'État, défini ici comme lieu politique de «condensation» des rapports sociaux de domination de classe $^{4}$. En effet, dans la mesure où une formation sociale - centrale ou coloniale - se structure de façon relativement autonome, on assiste à la constitution d'un lieu politique où se concentre, notamment, le pouvoir de sanctionner les infractions d'ordre "public», soit les comportements mettant en péril les rapports sociaux dominants à une époque donnée. Le point essentiel est que la structuration et la logique d'opération de ce lieu central de régulation des rapports sociaux sont déterminées par le mode de production que connait cette formation sociale ${ }^{5}$. C'est pour-

\footnotetext{
4 Cette définition opérationnelle, «dérivée» de celle de N. Poulantzas, L'État, le Pouvoir, le Socialisme (Paris, PUF, 1978), implique une distinction nécessaire entre l'État comme lieu politique, matérialisation d'un rapport de force et l'appareil spécifique de mise en oeuvre des politiques qui se développe à partir du $19 \mathrm{e}$ siècle surtout. Nous sommes bi:n conscient du caractère sommaire de cette définition, surtout en regard des remarquables développements qu'ont connus les recherches théoriques et empiriques sur l'État depuis dix ans. A ce propos, mentionnons à quel point la recherche historique sur l'Etat (à la fois comme forme politique de domination et comme appareil institutionnel) est déficiente au Canada et au Québec, surtout pour la période 1791-1930.

Quelques remarques s'imposent ici: le bref développement qui va suivre a pour seule ambition de situer le cadre général sur lequel repose notre analyse du système répressif bascanadien. Est-il besoin de préciser qu'il n'est pas question, dans ce court article, de prétendre régler de façon définitive ce qui apparaît comme le problème central des recherches sur le BasCanada de 1760 à 1840, soit les conditions de transition au mode de production capitaliste. La position adoptée ici doit être vue comme une réinterprétation s'inscrivant dans le débat sur la transition au capitalisme en Occident, débat historiographique dont la seule bibliographie couvrirait plusieurs dizaines de pages, et qui a comme principaux protagonistes M. Dobb, P. Sweezy, G. Bois, E. Kamenka, R. S. Neale, P. Anderson, P. Bezbakh, J. Banaji, R. Brenner, G. Hindess, P. Q. Hirst, C. Tilly, F. Braudel, M. Godelier, R. Hilton, K. Takashi, C. Hill, G. Lefebvre, A. Soboul, I. Wallerstein, E. Hobsbawm, E. P. Thompson, R. Robin, H. Medick, P. Kriedte, P. Vilar, C. Parain, G. Procacci, K. Polanyi, B. Moore, P. Corrigan, J. Foster, G. Rude, S. Jones et W. Kula. Qu'il suffise pour l'instant, de préciser que le qualificatif de «féodal», utilisé ici, ne doit pas être pris au sens restrictif que lui donne, par exemple R. Boutruche, Seigneurie et féodalité (Paris, Aubier, 1968), I: 20-25, mais comme caractérisant un mode de production basé notamment sur l'autonomie relative de la petite paysannerie et un mode spécifique (non-économique) d'ex-
} 
quoi on peut repérer, dans le continuum historique, une forme originale d'État qui caractérise les formations sociales occidentales jusqu'au milieu du 19e siècle: l'État féodal.

Ce modèle étatique est en fait constitué d'un ensemble disparate d'institutions permettant d'assurer le prélèvement du surplus social: institutions anciennes où la domination seigneuriale ou marchande sur les classes populaires se cristallisait (États généraux, parlements, bailliages, Église, franchises municipales) et par où passait le contrôle de la régulation; puis, de plus en plus importantes, institutions royales assumant, au niveau de la société globale, les fonctions centralisées indispensables au système féodal pleinement développé (justice, fiscalité, conduite de la guerre, régulation des colonies et protection du commerce extérieur). Ce rôle central de régulation assure, au sein de la formation sociale féodale, le contrôle en dernière instance des crises majeures du système. Tout se passe comme si, au-delà d'une marge implicite d'autonomie consentie aux masses populaires, se mettait en branle un ensemble de mesures correctives rétablissant les équilibres un instant menacés.

Le lieu d'initiation de ces mesures, de gestion de ces déséquilibres, est l'État. La légitimité de cet État, en système féodal, repose sur le respect de la balance subtile des rapports de force entre classes paysannes-artisanes et féodo-marchandes, inscrits formellement dans la coutume locale. Icci, la logique de régulation sociale ne passe pas prioritairement par l'État: elle repose avant tout sur la reproduction stable des équilibres locaux, institués dans la violence quotidienne des luttes de classe. Dans ce cadre, l'État est moins source normative que confluent, garantie dernière de préservation de la logique de reproduction féodale. C'est pourquoi, notamment, on assiste non pas à une fusion, mais à une superposition, par strates successives, d'appareils de répression divers ${ }^{6}$. Cependant la caractéristique majeure du système est que, dans la mesure où la cohésion interne des classes nobiliaire et marchande est suffisante, dans la mesure aussi où il n'existe pas de contradiction fondamentale entre le prélèvement féodal et la circulation marchande, on voit se concrétiser et se confirmer la décentralisation (ou déconcentration) des modes d'exercice du pouvoir.

En ce sens, le modèle anglais - soit les «libertés anglaises» matérialisées dans certaines institutions comme le jury, le juge de paix, l'organisation municipale et le pouvoir local de taxation, et consolidées par le compromis de 1688 - nous apparait non comme une «avancée»

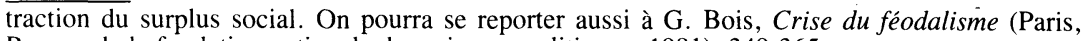
Presses de la fondation nationale des sciences politiques, 1981), 349-365.

6 Dans le cas de l'Angleterre, l'incroyable variété des cours de justice, l'extrême complexité des rapports entre corps policiers (constables, overseers, Bow Street Runners, police des ports, etc.), la multiplication anarchique des délits au Code pénal ne sont que des exemples caricaturaux de ce phénomène. Voir Léon Radzinowicz, A History of English Criminal Law and its Administration (Londres, Stevens \& Sons, 1948-68), 4 vols. 
capitaliste, mais comme un mode particulièrement réussi (autant qu'exceptionnel...) de régulation féodale. Or, c'est ce modèle que, dès la Conquête, l'Angleterre tentera de mettre en place au Canada. La conjoncture spécifique d'implantation de l'État colonial à la fin du $18 \mathrm{e}$ siècle ${ }^{7}$ fera que la décentralisation des pouvoirs de régulation sera compromise. Les facteurs qui, en système colonial, contribuent à minimiser l'emprise de l'État ${ }^{8}$ sont encore accentués, ici, par le caractère étranger d'une bonne partie de l'élite dirigeante. Ainsi, à la savante gradation des pôles d'autorité typique de modèle anglais (monarchie, gouvernement, juge de paix, pouvoir local) se substitue, dans la colonie, un isolement relatif du pouvoir central. Ce dysfonctionnement de l'autorité étatique sera encore accentué par les contradictions qui apparaissent au sein même de l'État colonial ${ }^{9}$. De ce fait, si l'on assiste à l'implantation formelle d'un État féodal de type colonial ${ }^{10}$ au Canada, les conditions spécifiques à cette formation sociale rendront encore plus aléatoire l'établissement effectif de l'autorité centrale, notamment en matière pénale.

\section{L'implantation du droit criminel}

Historiquement, la répression de l'illégalité dans toute formation sociale s'inscrit dans un cadre normatif donné. Cependant, les conditions d'apparition et de reproduction de ce cadre normatif diffèrent profondément selon la logique dominante d'organisation sociale à l'oeuvre. Ainsi, c'est par un glissement sémantique non dénué d'ambiguïté qu'on

\footnotetext{
7 Sur ce problème complexe, nous nous permettons de renvoyer à notre thèse: J.-M. Fecteau, op. cit., 37-55. Voir aussi l'analyse de P. Tousignant, «Problématique pour une nouvelle approche de la Constitution de 1791», Revue d'histoire de l'Amérique française, 27,2 (septembre 1973): 181-234.

8 Mentionnons, entre autres, l'immensité du territoire, les carences de l'infrastructure économique et l'accès relativement aisé à la terre et aux moyens de production.

9 Ce sont ces différents phénomènes qui forment les conditions fondamentales d'apparition de la lutte des Patriotes.

${ }_{10}$ La répartition des dépenses publiques de cet État est révélatrice du mode de régulation que l'on vient de décrire brièvement. Voici, par exemple, les dépenses du gouvernement civil du Bas-Canada du ler novembre 1820 au ler novembre 1821, par secteurs:

- Fonctionnement interne de l'État (Gouverneur,

Conseil exécutif, Assemblées)

$£ 25824(37 \%)$

- Entretien et reproduction de l'appareil étatique

(édifices publics, collecte du revenu, papeterie)

- Appareil juridique et répressif (Cours, police, prisons, milice)

- Régulation sociale préventive (aide, allocations, pensions, écoles, santé)

- Services publics d'infrastructure (inspection

générale, routes, postes, agriculture, pilotes)

£ $6819(10 \%)$

$£ 21996(31 \%)$

$£ 9899(14 \%)$

TOTAL

$£ 5340(8 \%)$

$£ 69878$
}

Source: Journal de la Chambre d'assemblée du Bas-Canada, 1821-2, appendice C.

On notera que $78 \%$ du budget gouvernemental (dépenses militaires exclues) concerne l'administration judiciaire $(31 \%)$ et les services internes à l'appareil d'État $(47 \%)$. La comparaison entre la part accordée aux salaires, aux dépenses d'infrastructure et à l'organisation des services est aussi fort intéressante. 
est amené à parler, à l'époque féodale, de «droit criminel». Car, contrairement au système actuel, ce droit n'est aucunement le produit d'une instance centrale de régulation - soit l'État - qui puisse s'appuyer sur la légitimité produite par l'idéologie de la "volonté collective». Tout au contraire, la base du droit féodal repose sur des compromis locaux peu à peu structurés dans un ensemble normatif appelé «coutume». Le pouvoir étatique féodal ne fait, en dernière analyse, que codifier ces coutumes et suppléer, par le biais des ordonnances royales - cas français ou de la législation - cas anglais - à leurs carences ou à leur désuétude. Le «droit criminel» féodal est donc constitué d'un noyau plus ou moins rigide de normes répressives dont le pouvoir royal s'est peu à peu - et fort précocement dans le cas de l'Angleterre - assuré le monopole d'opérationnalisation. En conséquence, on aurait tort de voir en lui un lourd appareil de sanction quadrillant étroitement l'organisation sociale. Il s'agit plutôt d'un instrument ultime d'arbitrage et de sanction auquel on ne fait appel qu'en dernier recours, et de façon tout à fait exceptionnelle. En ce sens, sa logique d'opération est étroitement reliée au type d'État que nous venons de décrire. La justice fait partie ainsi des attributs de la souveraineté royale; elle est par cela constitutive du pouvoir d'arbitrage en dernière instance qui est au fondement même de la logique étatique féodale.

Aussi, quand le roi d'Angleterre prend possession du Canada, son premier geste est-il d'introduire le corpus légal anglais en territoire conquis ${ }^{1}{ }^{1}$. Dans la mesure où l'introduction de ce cadre normatif spécifique touchait à la régulation interne des communautés locales, on devait s'attendre à de fortes résistances de la population soumise précédemment à la Coutume de Paris. Ces luttes en matière de droit civil dépassent notre champ d'analyse ${ }^{12}$. L'enjeu est cependant tout autre dans le cas du droit criminel. Dans l'idéologie féodale, les normes pénales concernent au premier chef la légitimité du souverain, directement visé par toute infraction à l'ordre immuable dont il est à la fois le produit et le gardien. C'est sur cette idéologie que s'appuie l'introduction du droit criminel anglais au Canada, d'abord par simple proclamation en 1763 puis, devant les critiques diverses soulevées par cette mesure ${ }^{13}$, par législation parlementaire en $1774^{14}$.

\footnotetext{
11 «We have given Power under our Great Seal to the Governors of our said Colonies respectively to erect and constitute, with the Advice of our said Councils respectively, Courts of Judicature and public Justice within our Said Colonies for hearing and determining all Causes, as well Criminal as Civil, according to Law and Equity, and as near as may be agreeable to the Laws of England.» Proclamation royale du 7 octobre 1763, A. Shortt et A. G. Doughty, Documents relatifs à l'histoire constitutionnelle du Canada (Ottawa, T. Mulvey, 1921), 138.

12 On pourra se référer à l'étude de $\mathrm{E}$. Kolish, Changements dans le droit privé au Québec et au Bas-Canada, entre 1760 et 1840. Attitude et réaction des contemporains, thèse de doctorat, Université de Montréal, $1980,758 \mathrm{p}$. 465 .

${ }_{13}$ Sur ce point de droit, voir J.-E. Côté, op. cit., 29-92 et A. Morel «La réception...», 453-

14 Acte de Québec, 14 Geo, III (1774), c. 83, art. 11.
} 
Pourtant, on a vu que le droit criminel, en système féodal, n'est pas, dans sa plus grande partie, le produit du pouvoir royal, même si celui-ci en assure le fonctionnement. On a une illustration frappante de ce phénomène dans le débat qui a présidé à l'introduction parlementaire des dispositions pénales anglaises au Canada. Rien, en principe, ne semblait s'opposer à un système où les normes pénales françaises eussent été administrées selon la procédure anglaise, sous l'autorité du roi d'Angleterre. Cela est si vrai que le solliciteur général d'Angleterre, Wedderburn, sous l'impulsion du secrétaire d'État lui-même, Lord Darmouth, proposera ce système hybride dans le second projet d'Acte de Québec, en 1774:

Et attendu que l'abolition de la torture et de ces cruels châtiments auxquels les habitants du Canada étaient autrefois exposés, et que l'introduction d'une loi plus douce et plus ferme dans les causes criminelles produirait d'excellents effets et que les Canadiens s'en rendent entièrement compte:

Qu'il soit décrété en vertu de l'autorité susdite que nul crime ou offense ne sera considéré comme haute trahison ou non-révélation d'attentat, dans la province de Québec et ses dépendances, à moins d'être reconnu comme haute trahison ou non- révélation de haute trahison en vertu des lois et statuts actuellement en vigueur dans la Grande-Bretagne, et que les dits statuts et lois seront appliqués et observés à tous égards dans tous les cas de haute trahison et non-révélation de haute trahison. Et qu'il soit de plus décrété qu'à l'égard de toutes les offenses pour lesquelles le coupable, en vertu des lois en vigueur au Canada, le 13 septembre 1759, pouvait être condamné à la peine de mort, l'accusé sera traduit en justice, acquitté ou condamné et puni conformément aux lois d'Angleterre sauf le cas où l'accusé déclaré coupable de quelque offense que ce soit, aura droit au bénéfice de clergie ${ }^{15}$, en vertu des lois d'Angleterre, alors il sera seulement passible d'une amende ou de l'emprisonnement ou requis de fournir une caution pour sa bonne conduite.

Et qu'il soit de plus statué que tout jugement, une fois le verdict rendu, ne pourra être suspendu sur des objections d'irrégularité dans l'acte d'accusation ou par suite de désistement dans la procédure. ${ }^{16}$

\footnotetext{
15 La revendication du bénéfice de clergé permet au condamné, dans certains cas, d'éviter la peine de mort dont il est passible.

16 «And Whereas the Abolition of the use of the torture and of those severe punishments to which the Inhabitants of Canada were formerly exposed and the Introduction of a more mild and certain Law in criminal cases would be highly beneficial to them and they are truly sensible of the same, Be it therefore Enacted by the Autority aforesaid that no Crimes of Offenses shall be High Treason or Misprision of Treason in the Province of Quebec and the dependencys thereof, But such as are High Treason or Misprision of High Treason by the Laws and Statutes now in force in Great Britain; and that the said Laws and Statutes shall be used and observed in Cases of High Treason and Misprision of High Treason in all respects whatsoever. And be it further Enacted That in regard to all other offences for which by the Laws in force in Canada and on the said 13th September 1759 the Offender was liable to suffer the pains of Death the party accused shall be tried and acquitted or condemned and punished according to the Laws of England. Provided
} 
La complexité de ce système rebutait le rédacteur du projet de loi lui-même et on résolut, finalement, d'introduire l'ensemble du droit criminel anglais «à l'égard de la définition et de la gravité de l'offense, du mode de poursuite et de procès, ainsi que des punitions et des amendes infligées par les dites lois à l'exclusion de toute autre règle de droit criminel ou mode de procédure à ce sujet qui a prévalu ou pu prévaloir dans la dite province, avant l'année de Notre-Seigneur mil sept cent soixante-quatre, nonobstant toute chose contraire à cette fin, contenue dans cet acte de quelque manière que ce soit» ${ }^{17}$.

En fait, les bouleversements qu'implique l'imposition du droit criminel anglais ne doivent pas être exagérés. Malgré les notables distinctions entre les modèles pénaux français et anglais ${ }^{18}$, les deux systèmes reposent sur une même logique répressive. Le changement brutal de cadre normatif menace surtout les classes qui, sous le Régime français, tiraient intérêt du caractère élitiste et relativement arbitraire de la procédure française, soit les seigneurs et les marchands français.

always that where by the Laws of England the benefit of Clergy is allowed upon any Conviction the Offender in such case shall only be fined and Imprisoned or bound to his good Behaviour, And Provided also That no Judgement shall after the Verdict given, be arrested upon any objection of Informality in the Indictment of Discontinuance in the Record.» A. Shortt et A. G. Doughty, op. cit., 524-525. Ces dispositions reflètent bien les vues du gouverneur colonial, Carleton: «The impossibility of abrogating at once the Laws of a Country well cultivated and long settled, and substituting others in their stead, become soon very apparent, the Manners and Habits and Sentiments of a people are so intimately connected with the Laws and the established form of things that it seems as easy to change at once the one as the other by any new and positive institution. (...) sound Policy has ever suggested, and Experience has confirmed it, as the most certain, and even expeditious Method of incorporating the Natives with the Conquering Nation, to impose very few, if any, of their own Laws and Customs upon the Conquered People, other than what were necessary to secure their Dependence and preserve and maintain the Conquest.»W. P. M. Kennedy et G. Lanctôt, Reports on the Laws of Quebec, 1767-1770 (Ottawa, F. A. Acland, 1931), 64,66 .

«As well in the Description and Quality of the Offense as in the Method of Prosecution and Trial; and the Punishments and Forfeitures thereby inflicted to the Exclusion of every other Rule of Criminal Law, or Mode of Proceeding thereon, which did or might prevail in the said Province before the Year of our Lord One thousand seven hundred and sixty-four; any Thing in this Act to the Contrary thereof in any Respect notwithstanding.» 14 Geo III (1774), c. 83, art. 11. Le préambule de l'article justifie ainsi cette décision: «And whereas the Certainty and Lenity of the Criminal Law of England, and the Benefits and Advantages resulting from the Use of it, have been sensibly felt by the Inhabitants, from an Experience of more than Nine Years, during which it has been uniformly administered...»

${ }_{18}$ Ces distinctions touchent deux aspects. En matière de procédure, le droit criminel français est de type «inquisitoire», et laisse au juge la détermination de la culpabilité; dans le cas -anglais, on est en présence d'une procédure «accusatoire», où la poursuite est menée par la victime et la culpabilité déterminée par un jury composé de pairs. En matière de peine, les lois criminelles anglaises forment un ensemble punitif particulièrement sévère (surnommé le «Code sanglant» par les contemporains): en 1800 , plus de 160 infractions criminelles sont passibles de la peine de mort sans bénéfice de clergé. Voir J. F. Stephen, A History of the Criminal Law of England (Londres, 1883), 3 vols; J. H. Baker, «Criminal Courts and Procedure at Common Law, 1550-1800», J. S. Cockburn (ed.), Crime in England, 1550-1800 (Londres, Methuen, 1977), 15-48. En France, au 18e siècle, on retrouve 27 cas pouvant conduire à la même sentence: Jean Imbert, La peine de mort. Histoire-Actualité (Paris, A. Colin, 1967). Sur la comparaison entre les systèmes de droit anglo-saxon et latin, voir l'intéressante étude de A. H. Pekelis, «Legal Techniques and Political Ideologies. A Comparative Study» dans R. Bendix (ed.), State and Society (Berkeley, University of California Press, 1973), 355-377. 
Caractéristique en ce sens est l'affaire Pélissier, qui survient en 1766. En décembre 1765, Jean Le Roy, marchand de Québec, est poursuivi pour voies de fait par une certaine Marie-Anne Béland, et se voit condamné à une amende de $£ 4$ et à donner caution de sa bonne conduite future. Un autre marchand, Christophe Pélissier, proteste contre cette sentence dans une lettre au gouverneur de la colonie. Sa critique est caractéristique du malaise des élites francophones devant la nouvelle procédure appliquée: «It is truly mortifying for a Man of Honour and spirit to submit in open court to Humiliations which he cannot possibly merit. ${ }^{19}$ Le brave marchand s'élève surtout contre le fait qu'un simple particulier, et qui plus est d'une classe inférieure, puisse traîner en cour un bourgeois prospère... Dans ce contexte, on ne s'étonnera pas de la virulente protestation qu'émet Chartier de Lotbinière à l'introduction statutaire du droit criminel anglais dans la colonie, par l'Acte de Québec de 1774:

Le Bill semble vouloir exprimer que c'est en grande partie, pour complaire aux désirs des Canadiens qu'on supprime dans leur païs toutes loix et manières de procéder pour le criminel à la françoise, et qu'on y substitue toutes les loix criminelles angloises et manières de procéder en conséquence; ce que je puis annoncer pour certain est que, dans la demande qu'ils font de leurs loix, il n'est nullement question d'en excepter celles qui regardent le criminel; et ils n'auroient pas manqué de l'exprimer, s'ils eussent préféré la loi angloise pour cette partie. On doit sentir que les lois civiles et les lois criminelles, étant formées et liées intimement l'une à l'autre par un même système, elles sont pour s'entreaider et s'appuier et réciproquement dans nombres de cas importans; qu'on ne sauroit conséquemment, de ce mélange de loix calculées sur des systèmes différens, espérer cette harmonie qui fait la baze de la sûreté et tranquillité publique, et qu'elles doivent nécessairement s'entrechoquer et s'affoiblir l'une l'autre d'instant en instant. ${ }^{20}$

Mais, dans les faits, malgré les inquiétudes des classes dominantes francophones ${ }^{21}$ et du premier choc culturel ressenti devant les nouvelles institutions ${ }^{22}$, l'introduction du droit criminel anglais n'eut certes pas, à la lumière de nos connaissances, l'impact qu'on serait tenté de lui

${ }^{19}$ D. Hay, op. cit., 93-94. Pélissier sera poursuivi pour libelle séditieux en janvier 1766. ANQ, T-011-0001/2248.

21 A. Shortt, et A. G. Doughty, op. cit., 547-548. tation articulée après 1774 . Il faudra attendre la radicalisation des luttes politiques entre l'Assemblée et le gouverneur pour voir ressurgir, dans un tout autre contexte, une critique du système judiciaire anglais. Voir, par exemple, Le Canadien des 25 et 30 mai 1832.

${ }_{22}$ Le cas de Marie-Josephte Corriveau, condamnée à mort pour meurtre en 1763, est passé à la légende. Il faut pourtant distinguer les diverses formes de résistance à l'envahisseur, retransmises par la mémoire collective, et le contact à long terme avec l'appareil répressif anglais. Sur la Corriveau, voir Luc Lacoursière, "Présence de la Corriveau», Cahiers des Dix, 38 (1973): 229264; E.-Z. Massicotte, «Les pendus encagés», Bulletin des recherches historiques, 37 (1931): 427-432. 
attribuer. A l'usage, les Canadiens surent rapidement s'adapter à ce système, dans la mesure surtout où les modalités d'insertion de l'appareil répressif dans le tissu des rapports sociaux de l'époque sont, on l'a vu, assez particulières. Tout au plus peut-on dire que, pour les masses populaires, la présence d'un appareil répressif étranger à leurs coutumes n'a fait qu'accentuer les conduites d'évitement déjà présentes sous le Régime français ${ }^{23}$.

\section{La structure de l'appareil répressif bas-canadien}

L'application du droit criminel repose sur un appareil juridique qui, au Canada, se stabilise à la fin du $18 \mathrm{e}$ siècle. Le système ne connâ̂tra, jusqu'au milieu du 19e siècle, que de légères altérations. Il s'agit, en fait, d'une copie plus ou moins fidèle des institutions anglaises, le législateur s'étant contenté d'apporter des modifications tenant compte des caractéristiques spécifiques de la colonie. Nous décrirons brièvement cette structure de mise en application du droit criminel.

Au niveau de l' «entrée» dans le système, l'arrestation des inculpés et des suspects fut d'abord confiée aux «baillis», élus par les propriétaires de chaque ville et village ${ }^{24}$. Cependant, devant le mauvais fonctionnement de cette mesure ${ }^{25}$, on reviendra, du moins dans les campagnes, à l'organisation mise en place sous le Régime français. Une série d'ordonnances et de lois ${ }^{26}$ redonne aux capitaines de milice le pouvoir de procéder à l'arrestation des délinquants dans les campagnes canadiennes. Dans les villes, une ordonnance de 1777 donne aux juges de paix la faculté de réglementer la police ${ }^{27}$, sans cependant leur confier explicitement celle de nommer des constables, comme c'est la pratique

23 Pour une opinion contraire, voir A. Morel, «La réception...», 480-509. Signaler, comme on le fait ici, la capacité d'adaptation des Canadiens français n'implique aucunement qu'il y ait eu «satisfaction» face à ce droit nouveau. Il est peu douteux que si on avait consulté l'habitant canadien, il aurait opté pour le système ancien avec lequel il avait appris, depuis un passé immémorial, à composer. Mais cette préférence induite, outre le fait qu'il eut été inconcevable qu'on ait pu favoriser son expression, ne doit pas nous amener à présumer un quelconque choc culturel ou, pire, un traumatisme profond supposément provoqué par un changement de système judiciaire. Sur l'impressionnante capacité de résistance idéologique et d'adaptation des masses paysannes en régime féodal, on pourra consulter l'intéressante étude de R. Hilton, «Feudalism in Europe: Problems for Historical Materialists», New Left Review, 147 (sept-oct 1984): 84-93.

24 «Ordonnance pour régler et établir les cours de justice, juges de paix, séances de quartier, baillis et autres matières touchant la distribution de la justice dans cette province», 17 septembre 1764. Les ordonnances des gouverneurs, de 1764 à 1791, ont été publiées dans les Rapports des archives publiques du Canada de 1913, 1914-5, et 1918.

25 Le secrétaire du gouverneur devra publier plusieurs avertissements enjoignant les propriétaires de procéder à l'élection prévue par la loi.

2617 Geo. III (1777), c. 5 et 8; 27 Geo. III (1787), c. 2 et $6 ; 34$ Geo. III (1794), c. 4; 36

Geo. III (1796), c. 11; 43 Geo. III (1803), c. 1 .
Le terme «police» est bien entendu pris ici au sens ancien, soit l'ensemble des mesures d'administration locale. 
en Angleterre ${ }^{28}$. Ce n'est qu'en 1787 qu'ils reçoivent le pouvoir de procéder à de telles nominations ${ }^{29}$. Notons en passant que les marguilliers de paroisse avaient aussi certains pouvoirs de police dans le cadre des différentes lois pourvoyant au maintien du bon ordre le dimanche ${ }^{30}$. Quant à la garde de nuit, ou «guêt», elle ne fut organisée qu'en $1818^{31}$.

La milice, les constables, les marguilliers et le guêt ne sont qu'un exemple de cette pratique de superposition éclectique des appareils de répression, caractéristique, on l'a vu, du mode de régulation féodale. Le système des cours manifeste la même tendance, mais de façon plus subtile. Jusqu'en 1794, les aléas de l'organisation judiciaire manifestent la perplexité des autorités coloniales face au système à adopter. L'ordonnance judiciaire du gouverneur Murray, en $1764^{32}$, met en place dans la colonie une copie à peu près conforme du système anglais. Après l'adoption de l'Acte de Québec, une ordonnance de 1777 reproduit presque intégralement l'organisation arrêtée en $1764^{33}$. Il faudra attendre 1794 pour qu'une solution originale soit adoptée ${ }^{34}$. Ainsi, en matière criminelle, on retrouve trois instances.

La cour du Banc du Roi, seule habilitée à prononcer une sentence de mort, et dont les juges sont nommés par le souverain, est le tribunal suprême de la colonie. Innovation remarquable par rapport à l'Angle-

${ }^{28} 17$ Geo. III (1777), c. 15. La nomination des constables, officiers de justice désignés en Angleterre par les juges de paix pour exécuter leurs mandats et maintenir l'ordre, relève du droit statutaire anglais. Dans le cas du Bas-Canada, un intéressant problème juridique se pose: en introduisant les lois criminelles anglaises dans la colonie, la mère patrie a-t-elle inclus dans ces dispositions l'organisation et les lois de police anglaises? Le fait que le gouvernement colonial a toute liberté de légiférer sur les matières de police implique-t-il, au contraire, que cet ensemble réglementaire doive faire l'objet d'une loi locale? Sur ce problème, on pourra consulter Philip C. Stenning, Le statut juridique de la police (Ottawa, ministère des Approvisionnements et Services, 1982), 7-60 (Commission de réforme du droit du Canada). Notons simplement, sur ce point, que nous n'avons retrouvé aucune trace de nomination de constables avant 1787 . Les officiers retrouvés sur les listes présentées à l'ouverture des sessions de la Cour du Banc du roi jusqu'à cette époque sont appelés «baillis», ce qui semble impliquer que l'ordonnance de 1764 était encore observée. En 1787, les marchands de Québec se plaignent que les magistrats de la ville, "n'ont pas de subalternes chargés de faire observer les lois, leurs injonctions manquent d'efficacité et sont impuissantes à contraindre le peuple à la soumission», A. Shortt et A. G. Doughty, op. cit., 893.

2927 Geo. III (1787), c. 6.

3048 Geo. III (1808), c. 26, continué jusqu'en 1813 par 52 Geo. III (1812), c. 6. Après une interruption de quatre ans, ces dispositions sont reprises par 57 Geo. III (1817), c. 3 et maintenues par plusieurs lois de portée temporaire jusqu'en 1840, quand 3-4 Vict. (1840), c. 6 les rend permanentes.

3158 Geo. III (1818), c. 2. Soulignons qu'il s'agit d'une force de police rémunérée, la charge de constable étant par contre une forme de service civil obligatoire.

32 «Ordonnance pour régler et établir les cours de justice...», 17 septembre 1764. Voir J. L'Heureux, op. cit., 266-331 et H. Neatby, op. cit., 298-319.

3317 Geo. III (1777), c. 5. Les instructions données à Carleton en 1775 laissaient pourtant au gouverneur une large marge de manoeuvre. «Instructions» à Carleton, 3 janvier 1775, RAPC, 1904, appendice E, 44

34 Geo. III (1794), c. 6. 
terre, on établit deux cours du Banc du Roi au Bas-Canada (Montréal et Québec), indépendantes l'une de l'autre ${ }^{35}$.

La cour des sessions trimestrielles, pour sa part, a juridiction sur tous les délits non passibles de la peine de mort. Chaque district (Québec, Montréal, Trois-Rivières et Gaspé) est doté d'une telle cour, tenue par les juges de paix nommés par le gouverneur. Les différentes cours trimestrielles forment une des institutions les plus importantes de la colonie: elles assurent en effet, en plus de leurs fonctions proprement judiciaires, l'administration locale des villes, et surveillent le travail des inspecteurs des chemins dans tout le district sous leur juridiction ${ }^{36}$.

Les deux instances judiciaires que l'on vient de décrire brièvement assurent l'essentiel des poursuites criminelles et respectent la procédure pénale traditionnelle (procès par jury, actes d'accusation formels, etc.) Il en est tout autrement des sessions hebdomadaires, mises en place dès 1764 et confirmées en 1794: il s'agit de tribunaux où deux juges de paix exercent une juridiction sommaire (avec le plus souvent appel aux cours trimestrielles) en matière de police locale ${ }^{37}$.

La structuration de ces cours de justice ne correspond aucunement à un système intégré d'instances de jugement se répartissant rigoureusement la sanction des crimes. Tout au contraire, chacune de ces cours a une historicité spécifique qui correspond à l'évolution de la monarchie anglaise. La cour du Banc du Roi est la plus ancienne et représente directement les attributions de justice du souverain par le biais de ses représentants. C'est pourquoi, notamment, le juge en chef est le personnage le plus important de la hiérarchie gouvernementale après le roi et le gouverneur, dans les colonies. Par contre, la cour des sessions trimestrielles a été créée statutairement au 14e siècle en Angleterre, et son origine est étroitement liée au contrôle des travailleurs ${ }^{38}$. Sa juridiction est limitée au comté ou aux districts au Bas-Canada; graduellement cependant, sa compétence, d'abord générale, a été réduite aux crimes relativement mineurs. En conséquence, tous les crimes sanctionnés par la Common Law ou les statuts anglais, dans la mesure où ils n'étaient pas passibles de la peine de mort, pouvaient être poursuivis

\footnotetext{
35 En Angleterre, l'unique cour du Banc du Roi tient des sessions itinérantes à périodes fixes à travers le pays. Il semble que la décision d'adopter ce système original au Bas-Canada ait tenu à l'étendue du pays et aux difficultés de transport. Ce système original avait été proposé dès 1769 par F. Maseres, procureur général de la colonie. Cf. Kennedy et Lanctôt, op. cit., 36.

36 Aussi étonnant que cela puisse paraître, aucune étude approfondie de cette instance n'a été menée à bien jusqu'à date, si l'on excepte l'importante analyse de Thierry Ruddel (à paraître). On pourra aussi consulter A. Drolet, La ville de Québec. Histoire municipale (Québec, la Société historique de Québec, 1967), vol. 2.

37 En Angleterre, les «sessions hebdomadaires» (ou «petty sessions») ne recevront de reconnaissance légale qu'au milieu du $19 \mathrm{e}$ siècle, même si la tendance à confier une juridiction sommaire se développe à partir des premières années du $18 \mathrm{e}$ siècle. Voir J. F. Stephen, op . cit., 1: $122-126$.

3818 Edw. III (1344), c. 2; 25 Edw. III (1350), c. 1 (Statute of Labourers); 34 Edw. III (1360), c. 1. J. F. Stephen, op. cit., 1: 111-116.
} 
indifféremment à la cour du Banc du Roi ou aux sessions trimestrielles ${ }^{39}$. Plus encore, il n'existait aucun appel des sessions au Banc du Roi ${ }^{40}$.

La procédure suivie dans ces deux dernières cours était, en vertu de l'Acte de Québec, la même que celle appliquée dans les cours britanniques. Cependant, diverses modifications furent apportées à cette procédure au Bas-Canada. Par exemple, les principaux officiers de justice étaient rémunérés par l'État, qui tentait de réduire au minimum la pratique des honoraires exigés des particuliers. Il en est ainsi des juges de la cour du Banc du Roi, du procureur général, des shérifs et des coroners, des greffiers de la Couronne, des geôliers et des bourreaux ${ }^{41}$. Sur un autre plan, on devra tenir compte de la langue parlée par l'accusé: en 1787, une ordonnance prévoit qu'au moins la moitié des membres du jury doivent parler la langue de l'accusé $e^{42}$; de plus, on s'assurera de la présence d'un interprète aux séances de la Cour. Cependant, la différence la plus fondamentale par rapport à la pratique anglaise, en matière de procédure, est certes le monopole de poursuite attribué au procureur général dans les causes criminelles. Alors qu'en Angleterre, l'immense majorité des poursuites sont le fait de particuliers ${ }^{43}$, c'est le contraire qui se produit au Canada. Dans la presque totalité des poursuites criminelles au Banc du Roi, de 1765 à 1840, c'est le procureur général qui prend en charge l'accusation ${ }^{44}$. Il s'agit d'un retour, dans la pratique sinon en théorie, au Régime français, autre témoignage du caractère particulièrement centralisé de l'administration de la justice en situation coloniale, provoquant un écart majeur par rapport à la logique d'opération du droit criminel anglais.

Même adapté aux spécificités coloniales, l'appareil judiciaire reflète bien le système légal qui, au cours des siècles, s'est mis en place autour

39 Au Bas-Canada, cette caractéristique provoquera des abus divers. Ainsi, en 1831, le comité des griefs de la Chambre d'Assemblée dénoncera la pratique du procureur général Stuart, qui poursuivait au niveau du Banc du Roi des crimes pouvant être jugés en sessions trimestrielles (les honoraires attribués dans les procès en cours du Banc du Roi étaient beaucoup plus élevés): Journal de la Chambre d'assemblée du Bas-Canada, 1831, appendice AA.

40 Les seuls moyens de retirer un procès de la juridiction des sessions trimestrielles étaient de requérir un mandat d'erreur («Writ of Error») ou de certoriari («Writ of Certoriari») d'un juge de la cour du Banc du Roi.

${ }_{41}$ Notons cependant qu'il ne s'agit que de salaires d'appoint permettant aux officiers d'exiger, dans certains cas, des honoraires. Par exemple, ce n'est qu'en 1830 que les geôliers se verront interdire de prélever des honoraires sur les prisonniers. Journal de la Chambre d'Assemblée du Bas-Canada, 1831-2, témoignage du 3 novembre 1831 .

${ }_{42} 27$ Geo. III (1787), c. 1. Contrairement à la coutume anglaise, les jurys, au Bas-Canada, sont choisis presqu'exclusivement dans les villes, et ce jusqu'en 1830

43 Voir D. Hay, «Private Prosecutor», Osgoode Hall Law Journal, 21 (1983): 165. Je tiens à remercier $\mathrm{A}$. Morel pour cette référence.

44 En 1839, le député Viger se plaint au secrétaire colonial, Lord Goderich, du procureur général James Stuart en ces termes: «Cet officier public a insisté avec un succès digne d'une meilleure cause, sur le droit absolument exclusif à l'espèce de monopole jusqu'à présent exercé par les procureurs généraux dans le pays, relativement aux poursuites criminelles, et de les porter et de les conduire seuls dans les cours, d'interdire à tout autre le droit de s'immiscer en aucune manière dans ces poursuites en qualité d'Avocat, représentant les accusateurs privés». D.-B. Viger à Lord Goderich, 10 novembre 1832, reproduite dans le Canadien, 23 janvier 1833. 
de la monarchie féodale anglaise. Un regard sur son fonctionnement concret nous permettra d'évaluer avec plus de précision sa place dans l'économie répressive de l'époque.

\section{L'appareil répressif face à la population criminelle: la gestion de l'infraction}

La description formelle qui vient d'être faite de la structure judiciaire peut, à première vue, donner l'impression d'un dense réseau institutionnel quadrillant le tissu social et y prélevant un échantillon plus ou moins représentatif des comportements déviants. Illusion de «modernité» du système qui disparait bien vite quand on analyse la pratique réelle des instances judiciaires ${ }^{45}$. Cette pratique peut être appréhendée sous trois aspects complémentaires, soit le recours aux tribunaux, la population-cible et le fonctionnement interne des instances judiciaires.

Le recours aux tribunaux en matière criminelle est une mesure d'exception dans les formations sociales de type féodal. Dans la mesure où la plupart des cas parviennent aux tribunaux sur plainte des particuliers devant un juge de paix, l'appareil judiciaire apparaît, en dernière analyse, beaucoup plus comme une instance ultime d'arbitrage ou de répression que comme le lieu privilégié d'aboutissement des délits. Ainsi, à titre indicatif, nous avons pu reconstituer assez fidèlement le nombre total de peines prononcées par l'ensemble des instances judiciaires du district de Québec, de juillet à septembre $1810^{46}$ :

Banc du Roi

Sessions trimestrielles

Sessions hebdomadaires

TOTAL
9 (crimes divers)

42 (crimes mineurs)

68 (infractions de police)

45 L'analyse qui va suivre s'appuie notamment sur le dépouillement systématique et la mise sur fiche de 2640 dossiers de la cour du Banc du Roi du district de Québec, pour la période allant de 1765 à 1840 inclusivement (ANQ, T-011-0001/2248-2270). Nous tenons à remercier M. Louis de Gonzalve Papillon, qui nous a permis de consulter ces documents à l'époque où ils étaient entreposés au ministère de la Justice. Les divers rapports des activités des cours publiés dans les journaux de la colonie de 1791 à 1840 ont aussi été utilisés (la Gazette de Québec, le Canadien, l'Aurore, le British American Register, le Courrier de Québec, et certains numéros de la Minerve, de la Montreal Gazette et du Montreal Herald). Nous avons aussi, avec Douglas Hay, fait un (trop) bref inventaire des documents judiciaires aux ANQ de Montréal. Qu'il nous soit permis ici d'insister sur l'incroyable richesse que recèlent les archives judiciaires pour l'histoire sociale des classes populaires de l'époque, richesse qui n'a d'équivalent que l'état déplorable dans lequel ces documents sont maintenus à Montréal.

${ }_{46}$ Données tirées de la Gazette de Québec et des archives judiciaires. Ces chiffres sont probablement légèrement inférieurs à la réalité. Par contre, le nombre de sentences prononcées en sessions de quartier est largement supérieur à la moyenne. Soulignons que l'absence de registre pour la période sous analyse rend difficile la construction de séries fiables pour cette juridiction. 
A l'époque, le district comptait environ 100000 habitants ${ }^{47}$. Le caractère marginal de la répression judiciaire dans le modèle de régulation féodal est de plus en plus souligné par les historiens ${ }^{48}$. Il est dû, certes, aux déficiences de l'appareil de prise en charge de la délinquance en première ligne (constables et miliciens) ${ }^{49}$, mais plus encore au fait que la plupart des litiges impliquant un délit criminel semblent bien être réglés au sein même de la communauté, notamment dans les campagnes $^{50}$. Dans le district de Québec, de 1791 à $1815,32 \%$ des accusés dont le lieu de domicile est mentionné provenaient de l'extérieur de la ville de Québec, alors que la proportion réelle des habitants du district demeurant à la campagne est de $85 \%$ environ. On retrouve dans les dossiers criminels quelques exemples de «composition» d'infractions criminelles, ententes hors cour qui ont, dans ce cas, évidemment échoué. Mais pour quelques arbitrages ratés de la part du seigneur, du curé, du notaire ou de l'avocat lui-même, combien de règlements à l'amiable entre familles, de compensations financières plus ou moins secrètes, de réputations sauvegardées et de fidélités garanties? A l'inverse, le recours à l'appareil répressif peut très bien être autant une vengeance personnelle, une humiliation publique délibérément planifiée, voire un moyen efficace de s'assurer une soumission, qu'une véritable recherche de justice ${ }^{51}$. Chose sûre, la justice du roi ne fait qu'effleurer le tissu social.

Il est en conséquence peu surprenant de constater que la «criminalité» sanctionnée par les cours est surtout urbaine. D'abord parce que

$47 \quad$ La tendance est encore plus forte en ce qui concerne le tribunal supérieur. De 1790 à 1799, pour l'ensemble du Bas-Canada (120 000 habitants environ), une moyenne annuelle de 25 personnes subit un procès devant la cour du Banc du Roi, si l'on s'en tient aux pièces retrouvées aux archives: ANQ, T-011-0001/2251-2252. Les documents trouvés à Québec permettent de constater que le phénomène devait s'appliquer à toute la province.

48 Voir, entre autres, Nicole Castan, Justice et répression en Languedoc à l'époque des Lumières (Paris, Flammarion, 1980); Michael Ignatieff, «State, Civil Society and Total Institutions: A Critique of Recent Social Histories of Punishment», Crime \& Justice, 3 (1981): 153-192; Alfred Soman, «Deviance and Criminal Justice in Western Europe, 1300-1800: An Essay in Structure», Criminal Justice History, 1 (1980): 3-28.

49 Personnel insuffisant (de 30 à 40 constables pour la ville de Québec au début du 19e siècle, assurant leur service tout en vaquant à leurs occupations journalières...), manque de formation, ignorance de la loi, équipement déficient, etc... Le récit des mésaventures des forces de l'ordre au Bas-Canada donnerait à lui seul matière à un article!

50 «Une négligence à découvrir les délinquants et à les amener en justice, domine universellement dans les campagnes éloignées de la ville, et tend à encourager la répétition des offenses», la Gazette de Québec, 9 août 1810 . Notons au passage que toutes les infractions criminelles faisant l'objet de plaintes formelles, qu'elles se produisent en campagne ou à la ville, aboutissent aux tribunaux urbains. Il est bon de rappeler que notre analyse porte exclusivement sur la gestion des crimes par l'appareil judiciaire, et non sur la criminalité «réelle» (le fameux «chiffre noir» des criminologues...); cette réserve émise, les conclusions énoncées ici s'appliquent donc tout aussi bien à la campagne qu'à la ville.

51 Nous avons calculé, quand les pièces au dossier le permettaient, quelle proportion des crimes poursuivis au Banc du Roi (district de Québec, période 1791-1815) concernaient un accusé et une victime qui, d'une façon ou d'une autre, se connaissaient déjà avant l'infraction. Sur les 83 procès où nous avons ce renseignement de façon certaine, 70 impliquaient une liaison victimeagresseur précédente au crime (soit $75 \%$ ). L'étroitesse de l'échantillon ne nous permet cependant pas d'arriver à une conclusion définitive sur ce point. 
l'afflux régulier d'étrangers dans les villes dépasse la capacité d'arbitrage interne de la communauté. Ensuite parce que c'est en ces lieux que l'insubordination des classes populaires, les occasions de défier les normes de soumission inscrites dans le modèle féodal sont les plus fréquentes. L'isolement relatif du pouvoir public en situation coloniale, que nous avons déjà souligné, prend ici toute sa dimension sociale. De multiples réseaux de sociabilité populaire se tissent, principalement autour d'une institution-type: l'auberge ou le cabaret, débits de boisson qui pullulent dans les villes, lieux de rencontre ou de divertissements divers où s'établissent des liens, se concoctent des projets plus ou moins licites, se ruminent de petites vengeances ou de grandes ambitions, s'élaborent des intrigues multiples. L'auberge est ce point de ralliement entre la population mouvante des migrants et des matelots et le petit peuple des apprentis et des serviteurs ${ }^{52}$; elle devient vite la cible sur laquelle se cristallisera la colère des élites bien-pensantes. Et pour cause: on est ici en présence d'un pôle de socialisation qui échappe, en bonne partie, à l'emprise des pouvoirs publics. Là s'arrêtent souvent le pouvoir du juge de paix, l'autorité du constable, la morale du prêtre et le paternalisme du notable. Les archives criminelles nous livrent parfois le tableau coloré des multiples transactions, des solidarités de circonstance qui s'y construisent.

S'il est donc une population-cible, il s'agit de ces catégories sociales, habituées des «lieux publics», plus ou moins en marge de la production, ou tout au moins en situation d'extrême dépendance. Ainsi les archives criminelles du district de Québec, pour la période 17911815 , nous renseignent avec certitude sur la position sociale des inculpés dans 81 cas $^{53}$ :

$\begin{array}{lr}\text { Soldats ou miliciens } & 22 \\ \text { Serviteurs et employés } & 20 \\ \text { Matelots } & 15 \\ \text { Prostituées, mendiants } & 3 \\ \text { Artisans } & 9 \\ \text { Indiens, Noirs } & 3 \\ \text { Habitants, cultivateurs } & 5 \\ \text { Marchands, professionnels } & 4 \\ \text { TOTAL } & 81\end{array}$

52 «Demandez aux pères et mères, informez-vous des maîtres et des maîtresses, de tous ceux qui sont obligés d'employer un grand nombre d'hommes à leurs services, quels sont les fruits que produisent ces asiles de dissipation et de fainéantise, où jusqu'à des enfants vont prendre sous le même toit des leçons et des exemples de la plus grossière et de la plus crapuleuse débauche, du mépris de toutes les règles de la morale comme de la bienséance, perdre tous les sentiments de l'honneur», L'Aurore, 29 novembre 1817.

53 La faiblesse de ce chiffre résulte du fait que nous avons dû renoncer à tenir compte du statut très vague («laborer», «spinster», «wife») accordé aux inculpés dans les actes d'accusation. Nous nous sommes donc rabattu sur les renseignements contenus dans les dépositions au procès, lorsque présentes au dossier. A moins que la conservation de ces pièces ne relève de critères tenant à la position sociale des inculpés, ce qui est fort douteux, il y a de bonnes chances pour que cet échantillon soit représentatif de l'ensemble des accusations. 
Le fait que Québec soit le principal port de la colonie et un important poste militaire - d'où la forte proportion de soldats et de matelots amenés devant la cour - est ici secondaire. Ce qui doit retenir avant tout notre attention, c'est le caractère «non représentatif» de cet échantillon par rapport à la démographie réelle de la ville de l'époque. On peut en dire autant du «monopole» quasi exclusif qu'exercent les hommes dans le tableau des accusés, et de l'importante proportion d'anglophones qui aboutissent au tribunal ${ }^{54}$, même si cette dernière tendance se stabilise relativement à partir de 1790. La justice royale, au Bas-Canada comme ailleurs, s'adresse à l'époque aux franges marginales et dépendantes des classes populaires. La faible activité de l'appareil judiciaire est aussi un témoignage du caractère relativement restreint du processus de dissolution des structures sociales d'ancien régime avant 1815, au BasCanada.

Plus encore, le fonctionnement interne des cours nous amène à penser qu'une procédure constante de filtrage des accusations est à l'oeuvre. Cette procédure a eu comme effet premier de maintenir à un niveau relativement constant la sanction punitive qu'exerce l'appareil de justice.

En effet, le cheminement qui conduit de la plainte à la condamnation est ponctué de multiples étapes qui contribuent à réduire encore le nombre de ceux qui subissent le poids de la peine: arrestation aléatoire du suspect, recherche difficile de témoins, examen du cas devant le grand jury, détermination de la culpabilité par le petit jury, et enfin délivrance possible du pardon royal après la sentence.

Le graphique II $^{55}$ nous montre cette procédure à l'oeuvre de 1791 à 1815 , pour le plus haut tribunal criminel de la colonie. On s'aperçoit que le petit jury a un rôle dominant dans le tamisage des accusations, permettant au rythme des inculpations de conserver une stabilité relative durant toute la période ${ }^{56}$. Enfin, le pardon royal vient réduire encore, mais cette fois après la sanction du crime, l'échelle de la punition effective ${ }^{57}$.

$54 \quad$ Voir le graphique I. A l'époque, la ville de Québec est à 75\% francophone.

55 Voir le graphique II. On trouvera les statistiques exactes à l'Annexe A.

56 La pondération des infractions effectuées par le petit jury est le contrepoids habituel à la férocité du droit criminel anglais. Devant la menace de mort qui pèse sur les personnes reconnues coupables des infractions les plus légères (par exemple, le vol d'objets d'une valeur de plus de 5 shillings dans une boutique), les jurys restent fort circonspects. Une autre pratique systématiquement utilisée est la sous-évaluation de l'importance du crime poursuivi (verdict partiel), notamment en affectant une valeur symbolique à l'objet du vol.

57 Nous avons dénombré 48 pardons entre 1775 et 1815, pour le seul district de Québec. Au Bas-Canada, le pardon est surtout utilisé pour commuer les peines de mort en bannissement, et plus rarement comme moyen de conciliation face aux révoltes populaires. Ainsi, en 1807, neuf habitants de St-Henri, condamnés pour rébellion contre l'Acte de Milice, reçoivent le pardon du gouverneur (ANQ, T-011-0001/2254). Pour une analyse fort intéressante de la logique d'opération du pardon royal, voir D. Hay, «Property, Authority and the Criminal Law», in D. Hay et al., Albion's Fatal Tree (New York, Pantheon, 1975), 40-49. 
RÉGULATION SOCIALE . . .

GRAPHIQUE I

COUR DU BANC DU ROI: DISTRICT DE QUEBEC

1775-1815

궁 $\vec{\pi}$ 융

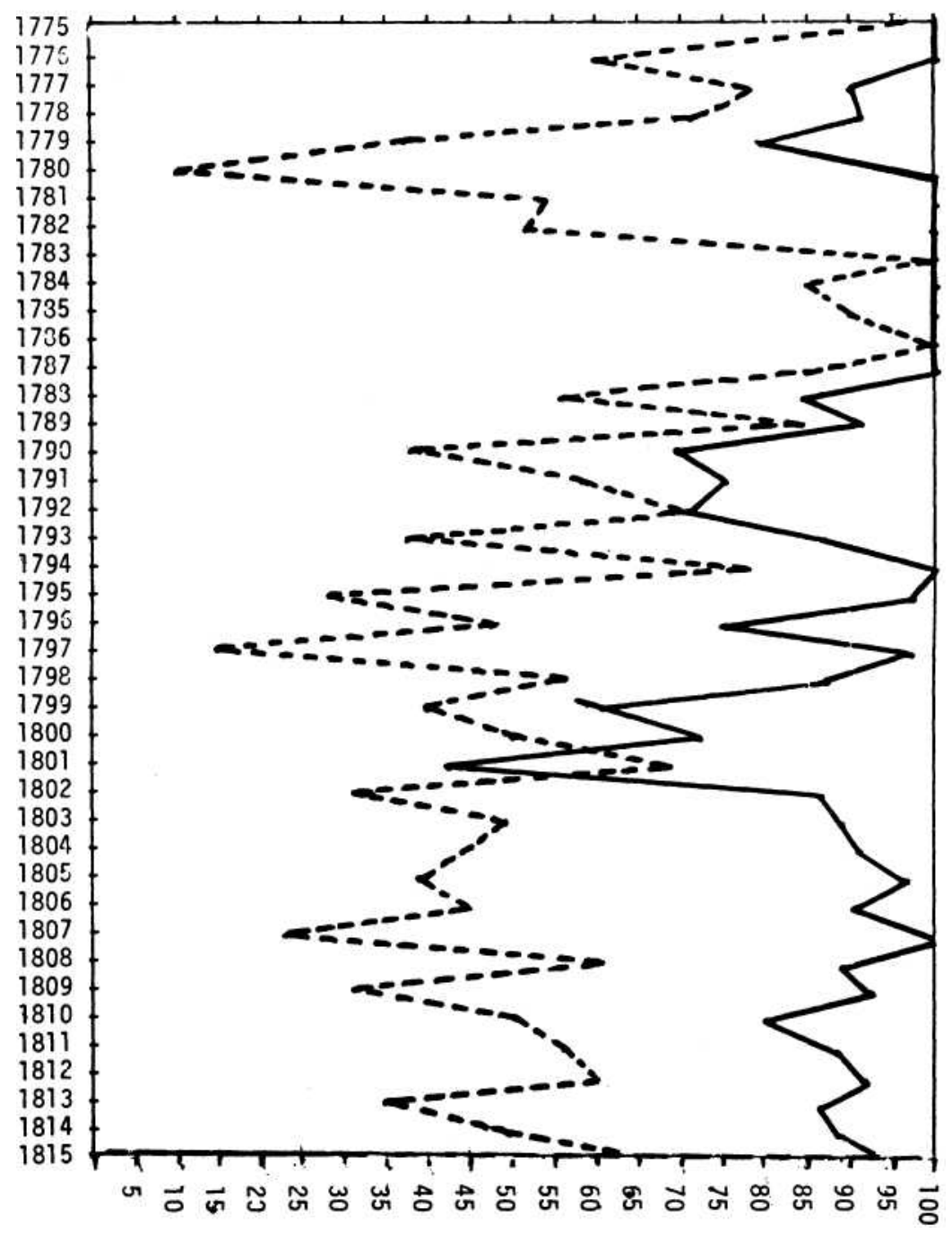

(-----) \% hommes/femmes

(........) \% anglophones/francophones 
GRAPHIQUE II

COUR DU BANC DU ROI : DISTRICT DE QUEBEC

$1775-1815$

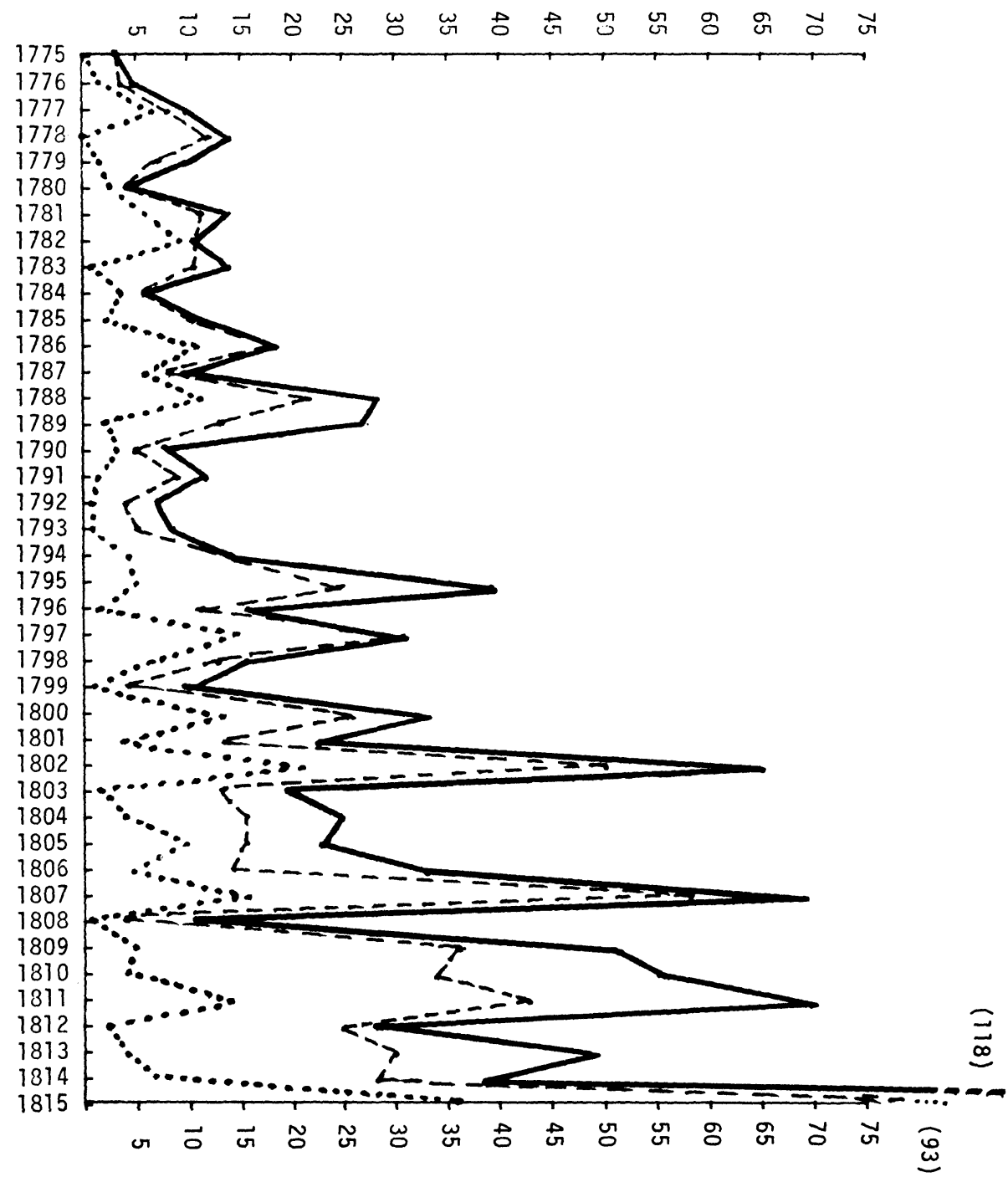

(-) Nombre de personnes accusees

(-.--) Accusations confirmees par le grand jury ("true bill")

$(\cdots \cdot)$ Verdicts de culpabilité du petit jury 
Manifestation royale du pouvoir de punir, l'appareil répressif entretient donc avec la gestion communautaire des infractions une relation de complémentarité. Le monopole de la sanction punitive des infractions que se réserve l'État doit donc être interprété ici à la lumière de la place réelle de l'appareil répressif dans la formation sociale. Il s'agit moins de «maintenir l'ordre» - ce qui est assuré par d'autres instances non immédiatement répressives, tels les rapports paternalistes d'autorité entre seigneurs et paysans, maîtres et apprentis, commerçants et commis - que de sélectionner, parmi les comportements délinquants, ceux qui, par leur gravité, leur fréquence ou, au contraire, leur caractère exceptionnel, échappent aux capacités locales de régulation. Plus précisément, c'est aux marges de la vie sociale qu'on retrouve présent le pouvoir royal de punir. Qu'elle vienne sanctionner le crime particulièrement atroce, apposer son châtiment exemplaire sur des infractions mineures mais trop répétées, arbitrer suprêmement un litige débordant les pratiques de conciliation de la communauté restreinte, ou marquer de sa violence soudaine et stochastique la population mouvante des villes, la justice du roi apparaît bien comme ce rempart ultime à l'impuissance temporaire, mais toujours récurrente, des élites locales. Cette logique d'opération se réflète immédiatement sur le type de sanction apportée aux infractions qui atteignent cette instance répressive.

\section{L'économie de la peine}

La férocité des peines prononcées par l'appareil répressif en système féodal est bien connue. L'utilisation systématique des peines afflictives, le recours fréquent au bannissement ou à la peine de mort dénotent un processus de répression exemplaire du corps que Foucault, parmi d'autres, a finement analysé ${ }^{58}$. Il est cependant important de bien repérer la logique à l'oeuvre derrière cette procédure répressive brutale. Cette analyse est d'autant plus importante que c'est essentiellement sur l'économie de la peine, et non sur l'organisation judiciaire en tant que telle, que portera l'offensive des humanistes et réformistes du $18 \mathrm{e}$ siècle. Nous nous contenterons, dans le cadre de cet article ${ }^{59}$, de mentionner que l'exemplarité des sentences du code criminel correspond à un objectif primordial: prévenir la répétition des infractions criminelles contre la personne et la propriété en stigmatisant de façon plus ou moins permanente la personne du délinquant. Une telle procédure impliquait cependant que l'appareil de sanction ne fonctionne que de façon exceptionnelle, sinon la brutalité de l'exemple risquait de se banaliser en violence administrative. C'est ce qui arrive, en Angleterre notamment,

\footnotetext{
9-71.

$58 \quad$ Michel Foucault, Surveiller et punir. Naissance de la prison (Paris, Gallimard, 1975),

59 On pourra consulter aussi sur ce point, J.-M. Fecteau, «Transition au capitalisme et régulation de la déviance. Quelques réflexions à partir du cas bas-canadien», dans Déviance et société, 8,4 (déc. 1984): 345-356.
} 
dans la première moitié du $18 \mathrm{e}$ siècle, alors que les élites locales, dominant le Parlement, pousseront à l'absurde ce système en multipliant les infractions passibles de la peine de mort: de 1688 à 1765 , le nombre de crimes pouvant aboutir à l'exécution passe de 50 à $160^{60}$. C'est contre cette «réaction parlementaire» que s'élèveront des réformateurs comme Fielding, Colquhoun, Howard et Bentham; ils s'attacheront à démontrer l'inefficacité de cette rigueur, et la nécessité de repenser l'économie des châtiments. La multiplication des échanges marchands, la commercialisation accélérée des rapports sociaux achèveront de condamner à terme les modes anciens de punir en les submergeant littéralement.

Alors se produit une cassure fondamentale dans le discours sur la peine: on en vient à distinguer implicitement d'une part les délits graves contre les personnes et les biens (vol avec violence, incendie volontaire, meurtre, viol, etc.), contre lequel le système traditionnel peut s'avérer efficace, et d'autre part l'infinie variété des infractions mineures (petits vols, vagabondage, mendicité, voies de fait, prostitution, rupture de service, etc.) contre lesquels le système est totalement impuissant. Ainsi naît l'idée d'un classement de la délinquance permettant un traitement différentiel des types de déviance. L'idéal d'une réforme du délinquant par le travail s'adresse essentiellement à la petite criminalité, et apparaît comme un mode nouveau de contrôle social s'appuyant sur la commission de l'infraction pour justifier une coercition disciplinaire sur les individus pris en faute. Derrière les réformes que propose Howard dans la gestion des prisons, il y a donc cette nécessité de séparer selon des critères précis la masse informelle des prisonniers. Et derrière la renaissance des célèbres «maisons de correction», il y a la volonté d'assigner un lieu privilégié au traitement de la petite délinquance.

Ce vent de réforme, qui obtient ses premiers résultats en Angleterre à la fin du $18 \mathrm{e}$ siècle, touche très tôt le Bas-Canada. Dès 1786, les magistrats de Québec demandent «que la ville de Québec soit dotée d'une maison de correction, d'une maison de détention et d'un hospice ${ }^{61}$. La distinction qui est faite ici est loin de n'être que sémantique: elle correspond à la césure faite entre le petit peuple des délinquants d'occasion et les auteurs de crimes graves qui n'ont que l'échafaud comme horizon. C'est dans ce contexte qu'apparaît, en 1799, l'«Acte qui pourvoit des maisons de correction dans les différents districts de cette province» ${ }^{62}$, une loi qui peut être considérée comme la première charte légale de l'idée de réforme pénale au Québec. Elle stipule que les fainéants, malfaiteurs et vagabonds seront enfermés et mis au travail

\footnotetext{
60 La plus tristement célèbre de ces lois criminelles est le «Black Act» de 1723 (9 Geo. I (1723), c. 22) qui, à lui seul, prévoit 350 cas précis où la peine de mort peut être imposée.

61 "Lettre des magistrats de Québec au Comité du Conseil sur le commerce et la police», 20 décembre 1786, A. Shortt et A. G. Doughty, op. cit., 909.

6239 Geo. III (1799), c. 6.
} 
dans des maisons de correction ${ }^{63}$. Ces institutions sont clairement conçues comme des centres de réforme du délinquant: on n'en tiendra pour preuve que cette extraordinaire disposition, ajoutée sur demande du Conseil législatif: «Pas plus de dix personnes seront détenues comme personnes fainéantes et déréglées, malfaiteurs et vagabonds, ou malfaiteurs incorrigibles en même temps dans aucune des maisons de correction constituées par le présent. ${ }^{64}{ }^{2}$ 'autre part, une autre limite apparaît dans le fait que ces institutions seront situées dans les prisons elles-mêmes. Elles auront cependant une administration autonome, assurée par trois juges de paix ${ }^{65}$.

Quant aux prisons proprement dites, les idées d'Howard trouveront une résonance au Bas-Canada dès le début du $19 \mathrm{e}$ siècle. L' "Acte des prisons» de 1815 pourvoit ainsi à l'érection de prisons à Québec et à Montréal ${ }^{66}$. Les districts de Gaspé (1808) et de Trois-Rivières (1811) feront l'objet de la même mesure ${ }^{67}$. La construction de ces prisons «modernes» ${ }^{68}$ ne doit cependant pas nous leurrer: elle a peu à voir avec la révolution pénitentiaire qui se manifestera vingt ans plus tard. On est plutôt en présence de la mise à jour, inspirée par les réformateurs anglais du $18 \mathrm{e}$ siècle, d'un instrument annexe de l'appareil judiciaire. Malgré la volonté de classement des prisonniers, la prison restera encore longtemps le réceptacle confus d'un peuple disparate: débiteurs insolvables, prévenus en attente de procès ou condamnés devant subir leur sentence à une date ultérieure, aliénés dont on ne sait que faire, individus en défaut de paiement d'amende, serviteurs punis par leur maittre, enfants de prisonniers sans autre refuge et même, pour une minorité, détenus purgeant une sentence de prison ${ }^{69}$. L'érection des nouvelles prisons ne

63 La loi va plus loin encore et permet même d'envisager le traitement par le travail des condamnés à des crimes graves, par voie de clémence de la part du juge ou du gouverneur. Il s'agit cependant d'une procédure exceptionnelle, qui démontre d'ailleurs implicitement l'existence de la distinction entre types de crime dont nous avons parlé plus haut.

64 «Not more than ten Persons Shall as idle and disorderly persons, rogues and vagabonds or incorrigible Rogues, be at any one time confined in either of the Houses of Correction hereby constituted.» 39 Geo. III (1799), c. 6, art. 2. Il semble que cette spécification ait été ajoutée par souci d'économie.

65 La prison commune était sous la responsabilité du shérif. Les renseignements que l'on détient sur le fonctionnement concret des maisons de correction nous confirment que ces établissements sont destinés en fait à la réforme des condamnés coupables d'infractions mineures. Ainsi, de 1802 à 1805 , les 125 prisonniers qui passent par la maison de correction de Montréal ont été reconnus coupables des crimes suivants: vagabondage, 55; désertion et apprentissage, 45; petit larcin, 8; voies de fait, 7; quête sans permission, 7; voies de fait et tentative de viol, 2; grand larcin, 1. Journal de la Chambre d'Assemblée du Bas-Canada, 1805, 460.

$6645 \mathrm{Geo}$. III (1805), c. 13. En fait, le projet date de quelques années auparavant, mais le problème que posait le financement de ces établissements retardera l'adoption de la loi. Sur ce point, voir J.-P. Wallot, Un Québec qui bougeait (Québec, Boréal Express, 1973), 47-106.

6748 Geo. III (1808), c. 35 et 51 Geo. III (1811), c. 17.

68 Le devis retenu pour la prison de Québec est inspiré d'Howard, Journal de la Chambre d'Assemblée du Bas-Canada, 1812, 123ss.

69 En 1816, 34\% seulement des prisonniers à la prison de Québec y étaient détenus en vertu d'une sentence de prison émise par la cour. Registre de la prison de Québec, 1816, ANQ, E$0017 / \mathrm{T}-1569$. 
fera que permettre une amorce de classement dans ce désordre. Elle permet aussi de compléter l'appareil répressif qui est mis en place au Bas-Canada $^{70}$.

\section{Conclusion}

Nous avons surtout insisté sur le caractère traditionnel du mode de répression qui s'organise dans le giron de l'État après la Conquête. Il faut, en effet, dans ce domaine comme dans bien d'autres, se débarrasser de l'image relativement «avant-gardiste» qu'on s'est plu à donner à l'administration britannique. L'Angleterre de l'époque n'est ni «capitaliste» et encore moins libérale ${ }^{71}$ : il s'agit simplement d'une société féodale particulièrement décentralisée, qui laisse aux élites locales («gentry» et marchands) un maximum d'autonomie dans la régulation des rapports sociaux. Son appareil de répression se ressent fortement de cette caractéristique spécifique et originale, et il faudra attendre les années 1825-1840 pour y dénoter des changements significatifs. En ce sens, la présence au Bas-Canada d'un mode traditionnel de gestion de la déviance ne doit aucunement s'interpréter en terme de «retard». Son peu d'emprise sur l'ensemble social est un attribut constitutif de sa logique d'opération. Tout au plus peut-on dire que ce manque structurel est encore aggravé en situation coloniale. Ici comme en métropole, on dénote cependant quelques tentatives de réforme - par la mise en place, notamment, des maisons de correction. Mais les véritables remises en question, la conscience aigüe de l'obsolescence rapide de l'économie répressive ancienne, ne se manifesteront de façon notable que vers 18151820 , et ce dans un contexte tout autre. C'est alors seulement qu'on pourra vraiment parler de transition à un mode nouveau de gestion des illégalismes.

\footnotetext{
70 Depuis la Conquête, on utilisait des casernes militaires ou des édifices publics désaffectés comme prisons.

71 Pour une vision tout à fait contraire, voir Gérald Bernier, «Sur quelques effets de la rupture structurelle engendrée par la conquête au Québec: 1760-1854», Revue d' histoire de l'Amérique française, 35,1 (juin 1981): 69-96. On pourra aussi se référer à $\mathrm{E}$. P. Thompson, Whigs and Hunters (New York, Pantheon, 1975).
} 
RÉGULATION SOCIALE . .

ANNEXE A

COUR DU BANC DU ROI: DISTRICT DE QUÉBEC 1775-1815

\begin{tabular}{|c|c|c|}
\hline & Accusés & $\begin{array}{c}\text { Grand } \\
\text { Jury }\end{array}$ \\
\hline 1775 & 3 & 3 \\
\hline 1776 & 5 & 4 \\
\hline 1777 & 10 & 8 \\
\hline 1778 & 14 & 13 \\
\hline 1779 & 10 & 7 \\
\hline 1780 & 4 & 4 \\
\hline 1781 & 14 & 12 \\
\hline 1782 & 11 & 11 \\
\hline 1783 & 14 & 11 \\
\hline 1784 & 7 & 7 \\
\hline 1785 & 12 & 11 \\
\hline 1786 & 18 & 18 \\
\hline 1787 & 10 & 9 \\
\hline 1788 & 27 & 22 \\
\hline 1789 & 26 & 13 \\
\hline 1790 & 8 & 5 \\
\hline 1791 & 12 & 9 \\
\hline 1792 & 7 & 4 \\
\hline 1793 & 8 & 5 \\
\hline 1794 & 14 & 14 \\
\hline 1795 & 39 & 25 \\
\hline 1796 & 16 & 12 \\
\hline 1797 & 31 & 31 \\
\hline 1798 & 16 & 13 \\
\hline 1799 & 10 & 4 \\
\hline 1800 & 33 & 26 \\
\hline 1801 & 23 & 13 \\
\hline 1802 & 65 & 50 \\
\hline 1803 & 20 & 13 \\
\hline 1804 & 25 & 16 \\
\hline 1805 & 23 & 16 \\
\hline 1806 & 33 & 14 \\
\hline 1807 & 69 & 59 \\
\hline 1808 & 11 & 4 \\
\hline 1809 & 52 & 36 \\
\hline 1810 & 56 & 34 \\
\hline 1811 & 70 & 43 \\
\hline 1812 & 29 & 25 \\
\hline 1813 & 49 & 30 \\
\hline 1814 & 39 & 28 \\
\hline 1815 & 118 & 93 \\
\hline
\end{tabular}

Remarque: Ces statistiques ont été établies, étant donné l'absence de registres, à partir des dossiers d'accusation et des comptes rendus publiés dans les journaux. Elles ne doivent donc être vues que comme indicatives d'une tendance. C'est le cas surtout pour les verdicts des petits jurys, que le greffier négligeait parfois de noter sur l'acte d'accusation. Ces carences documentaires nous empêchent donc d'établir avec certitude le degré exact de tamisage des accusations, sans pour autant que le phénomène lui-même soit discutable. 